\title{
Vereingte medizinische Gesellschaft in Kiew, Sektion für
} Augenheilkunde

\section{Sitzung am 8. Dezember 1928}

Vorsitzender: Prof. M. Lewitsky.

1. /. Tichonowitsch : Fall beiderseitiger Neuritis n. optici nachGrippe.

Aussprache: Wassütínsky erwähnt eine Zahl Originalarbeiten, die 1908 nach einer großen grippösen Epidemie erschienen. Drohender Beginn und verhältnismäßig günstiger Ausgang sprechen für die grippöse Atiologie. Lewitsky hatte einen Fall von Paraplegie und Optikusneuritis grippösen Ursprungs zuerst auf einem Auge, dann auf dem anderen, der vier Monate dauerte und ziemlich günstig endete.

2. /. Danilewsky: Exophthalmus bei Mucocele sinus ethmoidalis.

B., Bäuerin, 26 J. Vor einem halben Jahre erkrankte das linke Auge. St. praesens: Exophthalmus, das linke Auge ist lateralwärts und etwas nach unten verdrängt. Beim Betasten fühlt man einen Tumor von Taubeneigröße, der sich zwischen der hinteren Orbitalwand und dem Bulbus befindet. Die Geschwulst ist unbeweglich, elastisch und scheint mit der Orbitalwand $\bullet$ fest verbunden zu sein. Röntgen ohne Befund, Rw. negativ. Durch Operation und pathologische Unter-suchung ist festgestellt, daß in diesem Falle Mucocele osis ethmoidalis vorliegt. Nach der Operation verschwand der Exophthalmus und die Beschwerden und das Auge kamen zur Norm. 3. P. Powaliy-Klotschak: Zur Plastik des Konjunktivalsackes.

Demonstration von vier Kranken, bei welchen plastische Operationen nach Golowin, True, Panas und Duwerge-Welter ausgeführt wurden. In

200 Vereinigte medizinische Gesellschaft in Kiew.

zwei Fallen wurde Bildung des Konjunktivalsackes nach Panas-Golowin gemacht. Der Lappen dazu wurde von der Lippe genommen.

In einem Falle wurde die Operation nach Trucâ mit Wiederher-stellung des unteren Konjunktivalfornix gemacht; der Hautlappen ist von Stirn und Schläfen genommen worden. In diesem Falle folgte darauf eine ergänzende Operation: die Zilienwiederherstellung nach Spalth. Die Enderfolge waren befriedigende.

Der vierte Fall betrifft einen Kranken, dem die Wiederherstellung des Konjunktivalsackes nach Duverge-Welíer gemacht worden war. Einige Wochen nach der Operation begann das Zusammenschrumpfen des gebildeten Sackes, was eine zweite Operation (Kantotomie und Lippenschleimhauttransplantation) verlangte. Nach alien diesen plasti-schen Operationen wurde gleich die Prothese in den Sack eingeführt und die Lidränder zusammengenäht. Die Naht wurde auf drei Tage angelegt.

In alien Fallen regionäre Anästhesie.

Sítzung am 23. Dezember tg28. Vorsitzc nder: Prof. M. Lewitsky.

1. Priv.-Doz. A. Wassütinsky: Zur Frage liber die regionäre Anästhesie.

Übersichtsvortrag mit Angabe eigenen, unter Regionäranästhesie durchgeführten

Operationsmaterials. Auf Grund eigener Beobachtung und Literaturmaterials kommt W. zu dem Schluß, daß die regionäre Anästhesie eine sehr wertvolle Methode sei. 
2. I. Danihwsky: Fall extrabulbären Orbitalmelanosarkoms.Kranke N., 32 J. Vor einem halben Jahre ließ sie sich eine kleine

Geschwulst am medialen Winkel des rechten Auges entfernen. Zurzeit sieht man auf der Plica semilunaris und Caruncula lacrimaiis des rechten Auges eine erbsengroße Geschwulst von schwarzer Farbe. Unter der Lidhaut fühlt man eine höckerige Geschwulst, die mit der inneren Orbital-wand verbunden ist und sich in die Tiefe erstreckt. Auf der oberen und unteren Übergangsfalte der Konjunktiva sind kleine schwarze Par-tien, die allmählich nach außen zu verschwinden, zu bemerken. Der Bulbus etwas nach außen gedrängt, Augenbewegungen normal. Röntgeno-skopisch eine Verkleinerung der Sinus frontalis et maxillaris betreffenden Seite festgestellt. Vorgeschlagen: Röntgen- und Radiumbehandlung. 